\title{
Adaptive Threshold Determination Method for Recursive Maximum Filter
}

\author{
Ken'ichi Nishiguchi \\ Advanced Technology R\&D Center, Mitsubishi Electric Corporation \\ Tsukaguchi-Honmachi 8-1-1, Amagasaki-city, Hyogo, 661-8661 JAPAN \\ Nishiguchi.Kenichi@wrc.melco.co.jp
}

\begin{abstract}
The recursive maximum filter (RMF) is an algorithm proposed previously to detect dim moving targets in noisy image sequences. The detection of targets is performed by thresholding the output image of the RMF. In this paper, we propose a method to determine the threshold with a constant false alarm rate (CFAR) from the output image directly. The false alarm probability depends on the distribution of the output noise. In a transient state, the distribution of the output noise changes significantly. It is, however, difficult to predict time-varying distribution because of the nonlinearity in the RMF. Consequently, it is difficult to determine the threshold in advance, and some adaptive technique is required. We show that the mean and the variance of the output noise can be estimated from the output image directly, even when there are targets. The threshold with CFAR is obtained by using the estimates.
\end{abstract}

\section{Introduction}

Recently, there has been a growing interest in the detection of small low-contrast objects from optical or infrared (IR) image sequences [1]-[11]. The problem of detecting small low-contrast objects arises, for example, when we detect small moving targets from an on-board IR sensor [1, 2], and when we detect meteors, asteroids and satellites in the night sky from an array of charge coupled device (CCD) sensors on the focal plane of a. telescope $[3,4,5]$.

In these optical or IR sensor applications, the targets appear as points on the focal plane, so that pattern recognition techniques based on their shape are not available. Since the image data from these applications contains relatively high level noise compared with the intensity of the targets, thresholding in one frame does not yield the correct detection of the targets. In addition, the locations and trajectories of the targets are unknown, which makes frame integration techniques inefficient.
In order to overcome these difficulties, many detection methods have been proposed. Most of them process image sequences on the assumption that the targets move at constant velocities or with slow maneuvering. In $[6,7]$, a method using a 3-dimensional Fourier transform is proposed. In [3], a hypothesis test method is proposed, which stores every string of pixels that corresponds to all target trajectories in a lookup table of the tree structure. In $[1,2]$, a dynamic programming algorithm is applied to the problem as a track-beforedetect technique, and its performance is analyzed in $[8,9]$. These methods require an exhaustive processing time, which makes it difficult to process in real time, and they cannot treat the targets that do not move with nearly constant velocities.

The recursive maximum filter (RMF) is an algorithm proposed to detect dim moving targets with random movement in noisy image sequences $[10,11]$. The detection of targets is performed by thresholding the output image of the RMF. In this paper, we propose a method to determine the threshold with a constant false alarm rate (CFAR) from the output image directly. The false alarm probability is related to the distribution of the output noise, which is the output from the RMF when input images contain only noise. In a transient state, the mean and the variance of the output noise change significantly. It is, however, difficult to predict their actual levels because of the nonlinearity in the RMF. Consequently, it is difficult to determine the threshold in advance, and some adaptive technique is required. We show that the mean and variance of the output noise can be estimated from the output image directly, even when there are targets. The threshold with CFAR is obtained by using the estimates.

\section{Recursive Maximum Filter}

\subsection{Model of small target detection}

Let $Y_{i j}(k), i, j=1, \cdots, n, k=1,2, \cdots$ be the image sequence to be processed, where $(i, j)$ denotes the pixel 


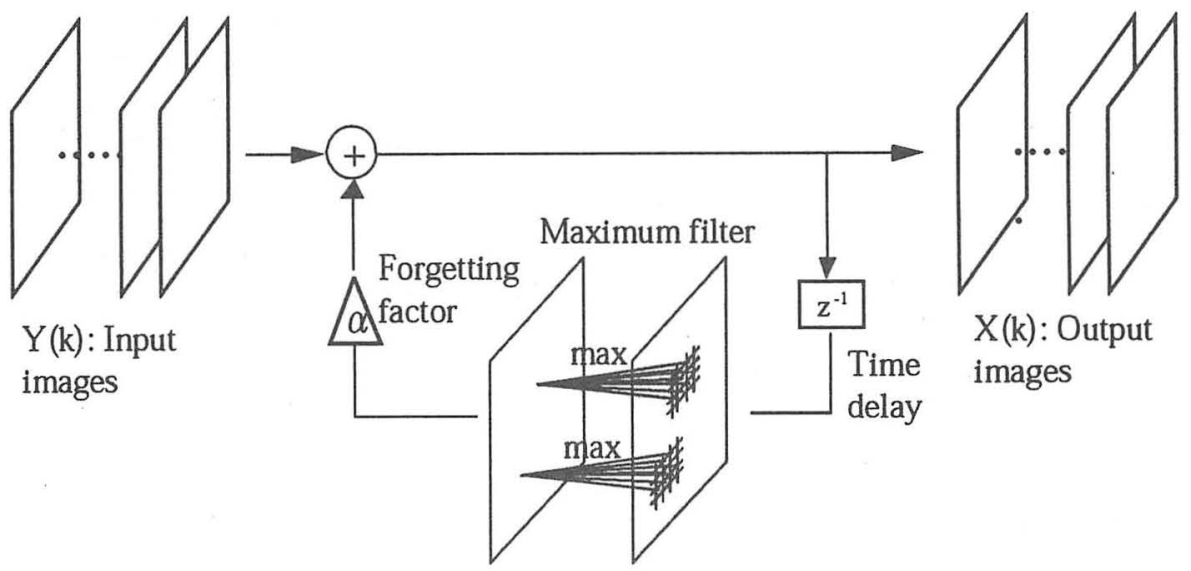

Fig. 1 Block diagram of recursive maximum filter.

(a) Original
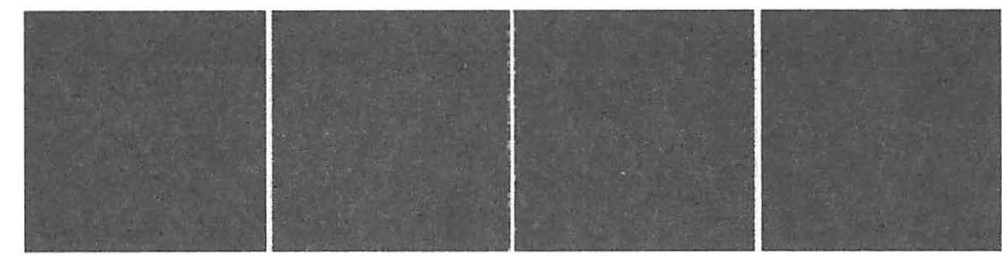

(b) Processed image

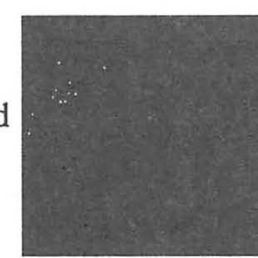

$\mathrm{k}=1$

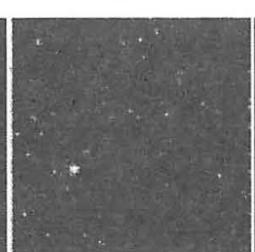

$\mathrm{k}=10$

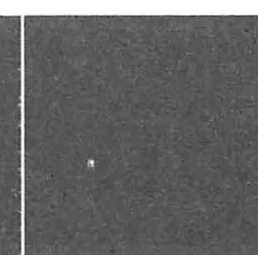

$\mathrm{k}=20$

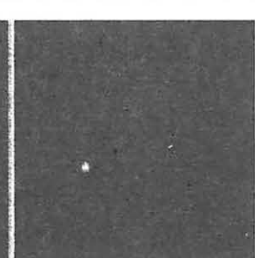

$\mathrm{k}=30$

Fig. 2 Dim target enhancement by the RMF.

number and $k$ the frame number at time $t_{k}$. The im age data contain targets, a structured background, clutter and noise. Here, we assume that the backgrounds except for noise are negligible or have already been whitened by pre-processing.

We assume that the targets move randomly from frame to frame. This assumption does not exclude the possibility of constant velocity movement, but implies a lack of knowledge about the motion of the targets. We also assume that the maximum velocity of a target is known, which is denoted by $v_{\max }$. For a pixel $(i, j)$, we define the neighborhood $D(i, j)$ as such a region that the target, which is present at the pixel $(i, j)$ in one image, may have existed in the preceding image. Let $\nu$ be the number of pixels contained in $D(i, j)$. Here $D(i, j)$ and $\nu$ are determined by the maximum velocity of the targets. For example, when $v_{\max } \leq 1$ pixels/frame, $D(i, j)$ is given by

$$
D(i, j)=\left\{\left(i^{\prime}, j^{\prime}\right) ; i^{\prime}=i, i \pm 1, j^{\prime}=j, j \pm 1\right\}
$$

and $\nu=9$.
We assume that the background noise $W_{i, j}(k)$ is Gaussian with a mean of $\mu_{\text {in }}$ and a variance of $\sigma_{\text {in }}^{2}$, and it is white both in time and space. Let $A$ be the intensity of a target. Then, the image data are represented as

$$
Y_{i j}(k)= \begin{cases}A+W_{i j}(k), & \text { target is present, } \\ W_{i j}(k), & \text { otherwise. }\end{cases}
$$

We define the input signal-to-noise ratio (SNR) of a target as

$$
\mathrm{SNR}_{\text {in }}=\frac{A}{\sigma_{\text {in }}} .
$$

If we detect targets in a single frame of image data by thresholding, then $\mathrm{SNR}_{\text {in }}$ must be greater than 6 or 7 .

The problem treated in this paper is the detection of targets that cannot be detected in a single frame of image data. Therefore, the $\mathrm{SNR}_{\text {in }}$ value we are concerned with is, say, less than 3 or 4 .

\subsection{Algorithm of Recursive Maximum Filter}

The algorithm of the RMF is represented as [10]

$$
X_{i, j}(0)=0 \text {, }
$$




$$
\begin{gathered}
X_{i, j}(k)=Y_{i, j}(k)+\alpha \max _{\left(i^{\prime}, j^{\prime}\right) \in D(i, j)} X_{i^{\prime}, j^{\prime}}(k-1), \\
k=1,2, \cdots,
\end{gathered}
$$

where $X_{i, j}(k), k=0,1,2, \cdots$ is the output image sequence of the RMF, and $\alpha$ is a forgetting coefficient that takes a value somewhat less than 1 (for example 0.95 or 0.98 ) and has a function to avoid the divergence of the RMF with elapsed time.

The algorithm of (5) can be implemented by the architecture shown in Fig. 1. The RMF in Fig. 1 has a local maximum filter in its feedback loop. Targets are enhanced by the RMF with elapsed time and detected by thresholding with an adequate threshold; although the $R M F$ is very simple, it is very good at enhancing dim moving targets. In Fig. 2, we show an example of an RMF application, where the size of the target is one pixel and the $\mathrm{SNR}_{\text {in }}$ value is 3 , and we set $\nu=3 \times 3$ and $\alpha=0.95$. As is shown in the figure, the target, which is not recognizable from each input image, is enhanced by the RMF and can be recognized easily.

\section{Target detection}

\subsection{Detection by thresholding}

The target detection scheme adopted here is based on thresholding the output images of the RMF. More specifically, if there is a region in the RMF's output image whose intensities exceed a specified threshold, we determine that there exists a target and its position is at the pixel with the maximum intensity. It is desirable that the threshold is set to hold a CFAR. To that end, the relationship between the threshold level and the false alarm probability has to be clarified.

\subsection{Distribution of output noise}

The false alarm probability is the probability that the maximum intensity of the RMF's output image pixels exceeds a threshold in the absence of a target. To evaluate the false alarm probability, it is necessary to know the distribution of output image intensities from the RMF in the absence of a target, which we referred to as the output noise. However, since this distribution is that of the maximum of the correlated variables, as shown in (5), it is difficult to derive analytically. Therefore, we use the model of the mean and the variance of the output noise, which was obtained previously with the aid of simulation [11]. Here, for simplicity, we assume $\mu_{\text {in }}=0$ and $\sigma_{\text {in }}=1$, which does not lose generality. Then, the mean and the variance of the model are represented as

$$
\begin{aligned}
& \mu_{\text {out }}(k)=\alpha \frac{1-\alpha^{k-1}}{1-\alpha} \mu_{0}, \\
& \sigma_{\text {out }}^{2}(k)=1+\alpha^{2} \sqrt{\frac{1-\alpha^{2(k-1)}}{1-\alpha^{2}}} \sigma_{0}^{2},
\end{aligned}
$$

where $\mu_{0}$ and $\sigma_{0}$ are parameters that depend on $\nu$ and $\alpha$. The values of $\mu_{0}$ and $\sigma_{0}$ are shown in Fig. 3 .

In Fig. 4 we show an example of the simulation results and fitting curves by the model of (6) and (7). The fitting also gave good results in other examples.

\subsection{Relation between threshold level and false alarm probability}

Let $H(k)$ be the detection threshold. If we set

$$
X_{\max }(k)=\max _{i, j} X_{i, j}(k),
$$

then the false alarm probability is represented as

$$
P_{\mathrm{FA}}=\mathcal{P}\left(X_{\max }(k) \geq H(k) \mid \text { no target }\right),
$$

which has to be kept constant for CFAR detection. We normalize $X_{\max }(k)$ as

$$
\bar{X}_{\max }(k)=\frac{X_{\max }(k)-\mu_{\text {out }}(k)}{\sigma_{\text {out }}(k)},
$$

and assume that the distribution of $\bar{X}_{\max }(k)$ does not depend on $k$. This assumption holds approximately, which is verified by numerical simulation. If we set $h$ as the threshold for $\bar{X}_{\max }(k)$, then the false alarm probability given by

$$
P_{\mathrm{FA}}(h)=\mathcal{P}\left(\bar{X}_{\max }(k) \geq h \mid \text { no target }\right),
$$

does not depend on $k$. Therefore, CFAR detection is realized by setting the threshold as

$$
H(k)=\mu_{\text {out }}(k)+h \sigma_{\text {out }}(k),
$$

where $h$ is kept constant.

We obtained $10^{5}$ samples of $\bar{X}_{\max }$ by simulation for each pair of parameters $\nu$ and $\alpha$. The empirical probabilities of false alarm by the samples are shown in Fig. 5. As is shown in the figure, the $P_{\mathrm{FA}}$ curve depends on both $\nu$ and $\alpha$, but it has little dependency on $\nu$. From the figure, if we set $h=7.5$, then it is expected that $P_{\text {FA }}$ is less than $10^{-4}$ in all cases.

\section{Adaptive threshold determination}

\subsection{Difficulty in threshold determination}

In a previous section, we assumed that the mean and the variance of the input noise are $\mu_{\text {in }}=0$ and $\sigma_{\text {in }}=1$, respectively. In the general case, let $\tilde{Y}_{i, j}(k)$ and $\tilde{X}_{i, j}(k)$ be the input and output images of the RMF, respectively. The recursive algorithm of the RMF for those images is

$$
\begin{aligned}
& \tilde{X}_{i, j}(0)=0, \\
& \tilde{X}_{i, j}(k)=\tilde{Y}_{i, j}(k)+\alpha \max _{\left(i^{\prime}, j^{\prime}\right) \in D(i, j)} \tilde{X}_{i^{\prime} j^{\prime}}(k-1), \\
& \quad k=1,2, \cdots,
\end{aligned}
$$




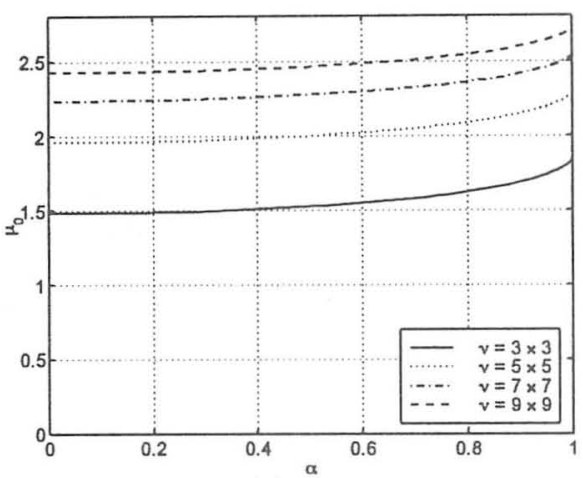

(a) $\mu_{0}$

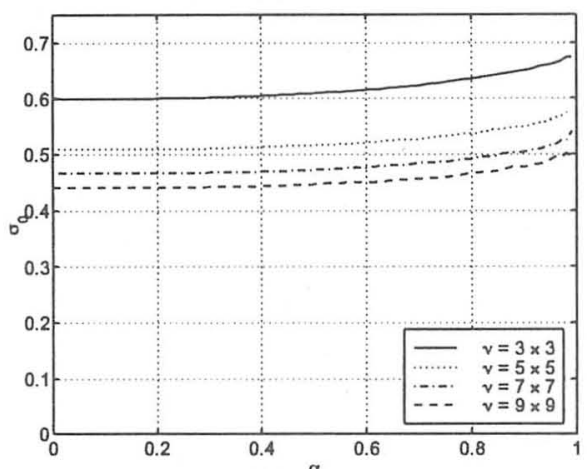

(b) $\stackrel{\alpha}{\sigma}$

Fig. 3 Estimate of model parameters of the output distribution when input contains only noise.

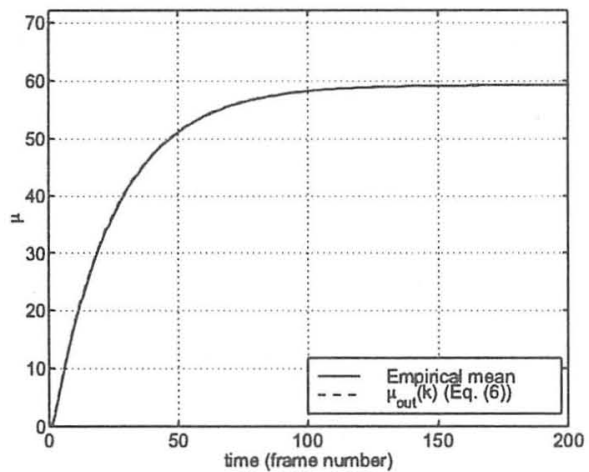

(a) Mean

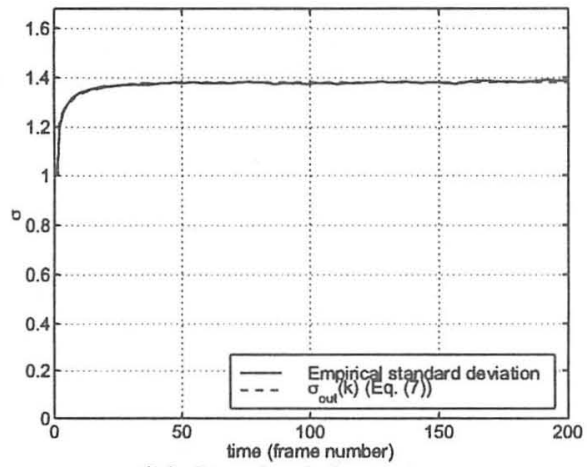

(b) Standard deviation

Fig. 4 Model fitting to the output distribution when input contains only noise.

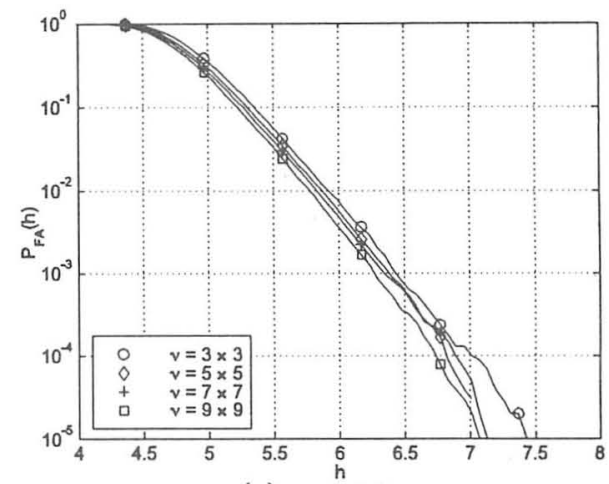

(a) $\alpha=0.9$

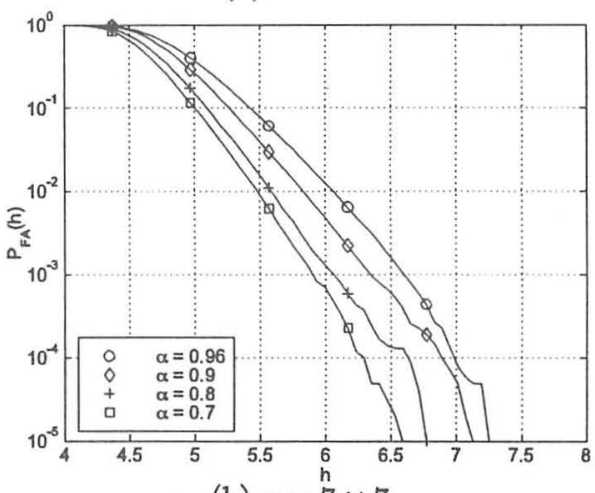

(b) $\nu=7 \times 7$

Fig. 5 False alarm probability.

As in the section 2, let $Y_{i, j}(k)$ and $X_{i, j}(k)$ be the input and output images of the RMF when the input noise has a mean of zero and a variance of one. We decompose the input and the output images as follows:

$$
\begin{aligned}
& \tilde{Y}_{i, j}(k)=Y_{i, j}(k) \sigma_{\text {in }}+\mu_{\text {in }}, \\
& \tilde{X}_{i, j}(k)=X_{i, j}(k) \sigma_{\text {in }}+\mu_{X}(k),
\end{aligned}
$$

where $\mu_{X}(k)$ is defined by (16). Substituting these representations into (13) and (14), and using (4) and (5) yields

$$
\begin{aligned}
& \mu_{X}(0)=0, \\
& \mu_{X}(k)=\mu_{\text {in }}+\alpha \mu_{X}(k-1), \quad k=1,2, \cdots,
\end{aligned}
$$

which can be solved to give

$$
\mu_{X}(k)=\frac{1-\alpha^{k}}{1-\alpha} \mu_{\text {in }} .
$$

From the above consideration, we derive the mean and the variance of the general output image $\tilde{X}_{i, j}(k)$ as

$$
\begin{aligned}
& \tilde{\mu}_{\text {out }}(k)=\mu_{\text {out }}(k) \sigma_{\text {in }}+\frac{1-\alpha^{k}}{1-\alpha} \mu_{\text {in }}, \\
& \tilde{\sigma}_{\text {out }}^{2}(k)=\sigma_{\text {out }}^{2}(k) \sigma_{\text {in }}^{2} .
\end{aligned}
$$

The threshold in the general case is written as

$$
H(k)=\tilde{\mu}_{\text {out }}(k)+h \tilde{\sigma}_{\text {out }}(k),
$$




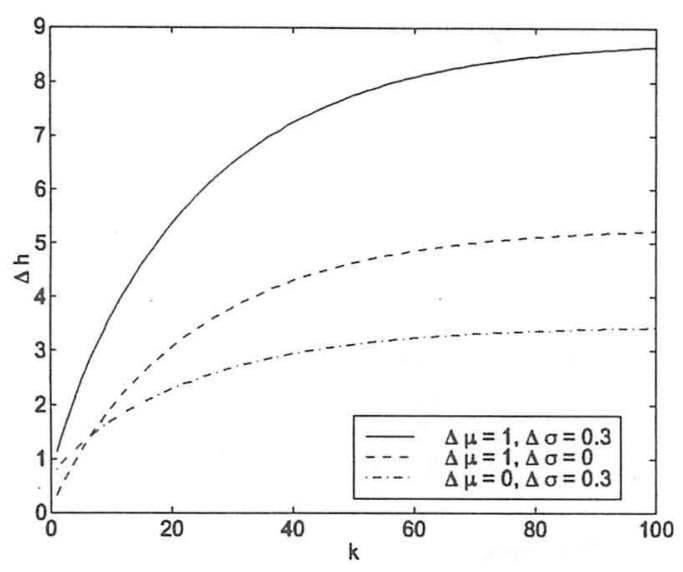

Fig. 6 Error of threshold parameter $\left(\sigma_{\text {in }}=3\right)$.

$$
=\left[\mu_{\mathrm{out}}(k)+h \sigma_{\mathrm{out}}(k)\right] \sigma_{\text {in }}+\frac{1-\alpha^{k}}{1-\alpha} \mu_{\text {in }} .
$$

If we know the accurate values of $\mu_{\text {in }}$ and $\sigma_{\text {in }}$, we can determine the threshold by this equation. In a practical situation, however, parameter values include measurement errors and may vary temporally. We suppose that we determine the threshold by using $\widehat{\mu}_{\text {in }}$ and $\widehat{\sigma}_{\text {in }}$ instead of $\mu_{\text {in }}$ and $\sigma_{\text {in }}$ as

$$
\widehat{H}(k)=\left[\mu_{\text {out }}(k)+h \sigma_{\text {out }}(k)\right] \widehat{\sigma}_{\text {in }}+\frac{1-\alpha^{k}}{1-\alpha} \widehat{\mu}_{\text {in }} .
$$

Then, the effective value of the normalized threshold $h$ becomes

$$
\begin{aligned}
\widehat{h}= & \frac{\widehat{H}(k)-\tilde{\mu}_{\text {out }}(k)}{\tilde{\sigma}_{\text {out }}(k)} \\
= & \frac{\widehat{\sigma}_{\text {in }}}{\sigma_{\text {in }}} h+\frac{\mu_{\text {out }}(k)}{\sigma_{\text {out }}(k)} \frac{\widehat{\sigma}_{\text {in }}-\sigma_{\text {in }}}{\sigma_{\text {in }}} \\
& +\frac{1-\alpha^{k}}{(1-\alpha) \sigma_{\text {out }}(k)} \frac{\widehat{\mu}_{\text {in }}-\mu_{\text {in }}}{\sigma_{\text {in }}}
\end{aligned}
$$

and its error is

$$
\begin{aligned}
\Delta h & \equiv \widehat{h}-h \\
& \left.=\left(h+\frac{\mu_{\text {out }}(k)}{\sigma_{\text {out }}(k)}\right) \frac{\Delta \sigma}{\sigma}+\frac{1-\alpha^{k}}{1-\alpha} \frac{1}{\sigma_{\text {out }}(k}\right) \frac{\Delta \mu}{\sigma},
\end{aligned}
$$

where $\Delta \mu \equiv \widehat{\mu}-\mu$ and $\Delta \sigma \equiv \widehat{\sigma}-\sigma$ denote measurement errors. Figure 6 is an example of the error of the normalized threshold calculated by (25). Since this error has a tendency to expand with elapsed time, as shown in the figure, the determination of the adequate threshold in advance is no easy task.

\subsection{Proposed method}

If the mean and the variance of the output noise can be estimated from the output image directly, it is preferable to use them. Such direct estimation is possible for the RMF by its feature. In Fig. 7 (a)-(c) and Fig. 8 (a)-(c), examples of the histogram of intensities of output images are plotted. Figure 7 (a)-(c) is the case of no target; hence, these are equal to histograms of output noise. On the contrary, Fig. 8 is the case where a target exists. The difference between the histograms of the two cases is only the long tail on the right side, and the left sides of the peaks are the same. Using this property, we can estimate the mean and variance of the output noise. Concretely, we use the following procedure:

(1) Make a histogram of the output image, which is represented by $(x(\ell), F(\ell)), \ell=1, \cdots, \ell_{\max }$, where $x(\ell)$ denotes the location of the center of the $\ell$ 'th bin and $F(\ell)$ the number of pixels that are in the '’th bin.

(2) Find the mode $\ell^{*}$ by

$$
\ell^{*}=\arg \max _{\ell} F(\ell),
$$

and estimate the mean of the output noise by

$$
\widehat{\mu}_{\text {out }}=x\left(\ell^{*}\right) .
$$

(3) Calculate the estimate of the variance of the output noise by

$$
\widehat{\sigma}_{\text {out }}^{2}=\frac{\sum_{\ell=1}^{\ell^{*}-1}\left(x\left(\ell^{*}\right)-x(\ell)\right)^{2} F(\ell)}{\sum_{\ell=1}^{\ell^{*}-1} F(\ell)} .
$$

\subsection{Simulation results}

In Figs. 7(d) and 8(d), we show simulation results. Here, the image size is $256 \times 256$ pixels, and we set the parameters of the RMF as $\nu=5 \times 5$ and $\alpha=0.96$. Figure 7(d) is for the case of no target and Fig. 8(d) for the case where there exists a dim moving target. The amount of movement of the target was given by the mean $(1.25,0)$ pixels/frame and the standard deviation of $(0.75,0.75)$ pixels/frame. In Figs. $7(d)$ and $8(d)$, the estimates of $\mu_{\text {out }}$ and $H$ were obtained by the procedure of the last section. The true values of $\mu_{\text {out }}$ and $H$ were calculated using (6), (7) and (12). Comparing these two figures, we find that the thresholds of both cases are about the same and approximate the true one. In these figures, $X_{\max }$ is also plotted. As is shown in these figures, $X_{\max }$ does not exceed the threshold in the case of no target (Fig. 7(d)), while, in the case with a target (Fig. 8(d)), $X_{\max }$ increases with elapsed time and exceeds the threshold. In Fig. 9, the detection result by the adaptive threshold for the case of Fig. 2 is plotted. 


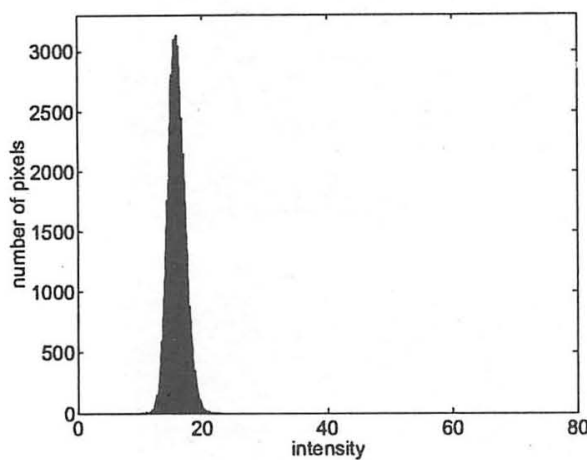

(a) Histogram, $k=10$

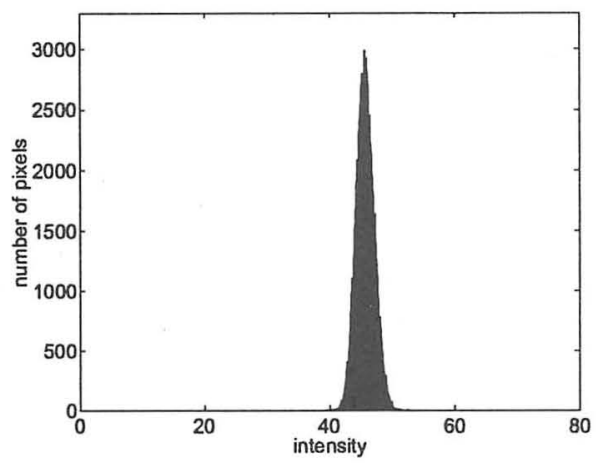

(b) Histogram, $k=50$

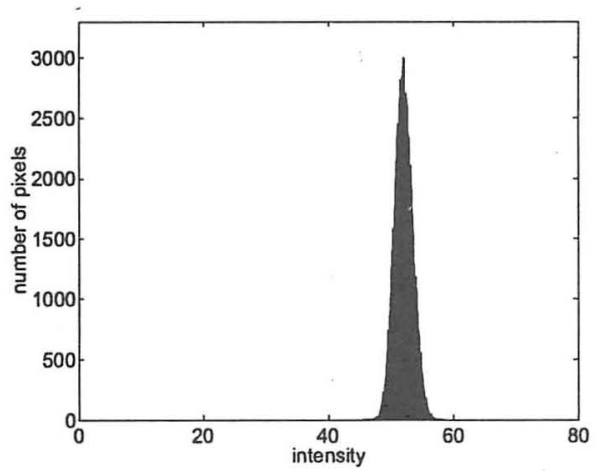

(c) Histogram, $k=100$

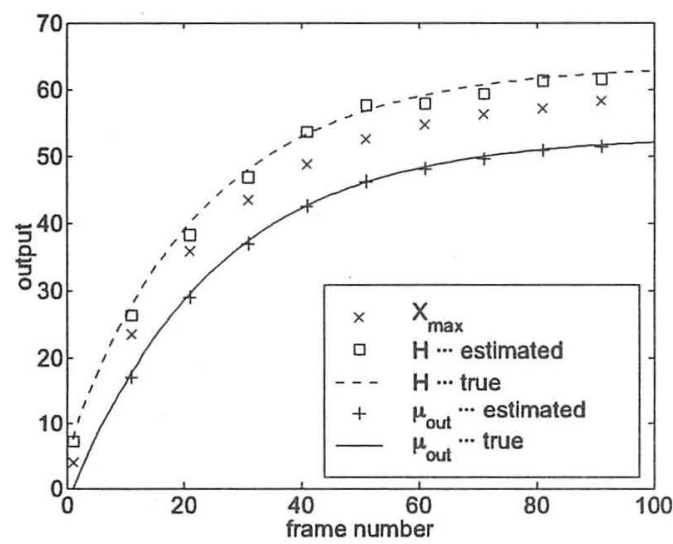

(d) Adaptive threshold

Fig. 7 Simulation result, no target.

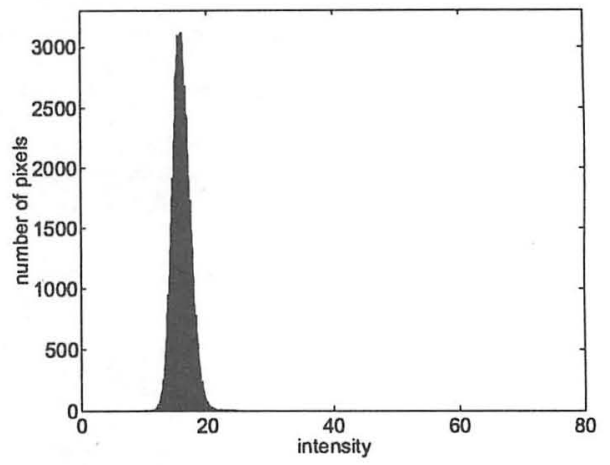

(a) Histogram, $k=10$

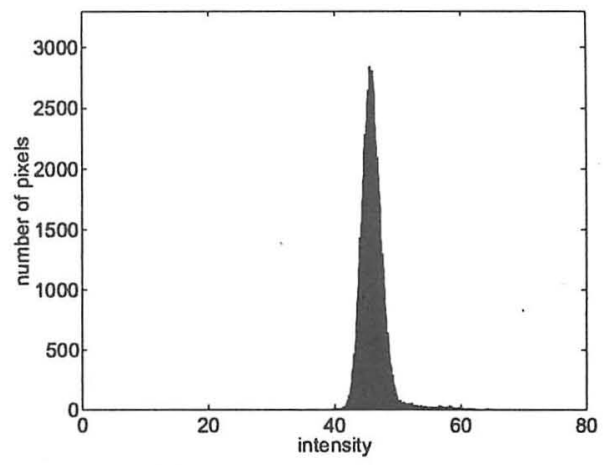

(b) Histogram, $k=50$

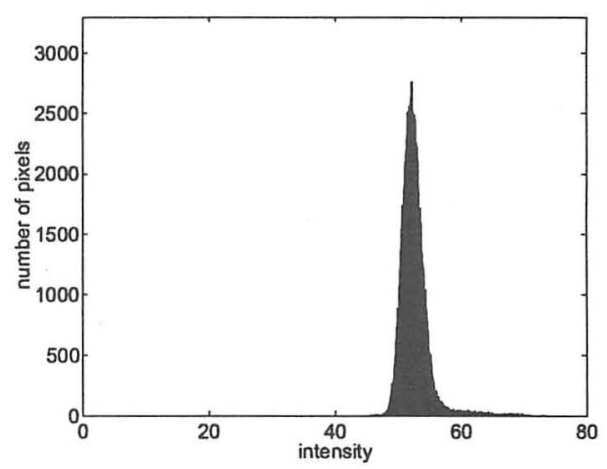

(c) Histogram, $k=100$

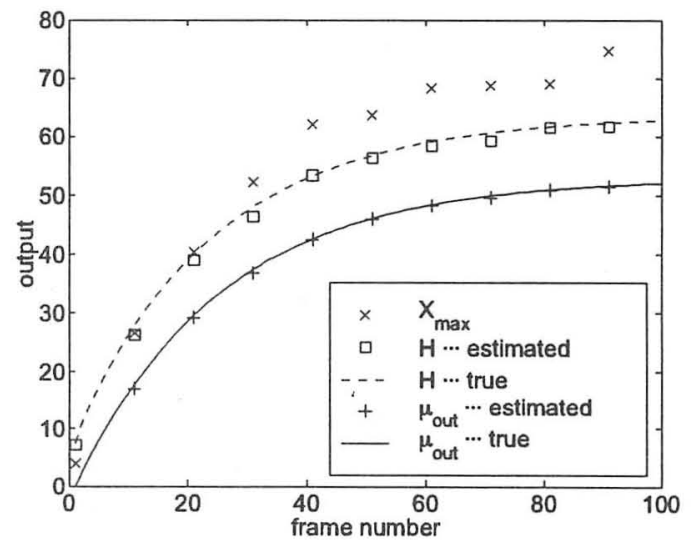

(d) Adaptive threshold

Fig. 8 Simulation result, target with SNR of 3 . 
(a) Processed image
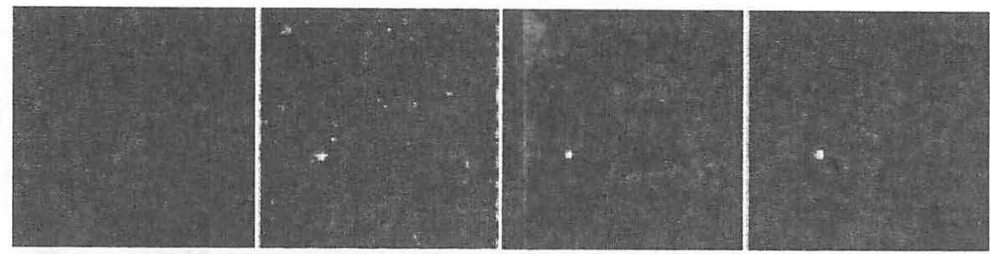

(b) Detection result

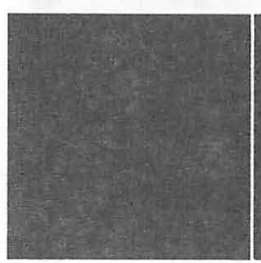

$\mathrm{k}=1$

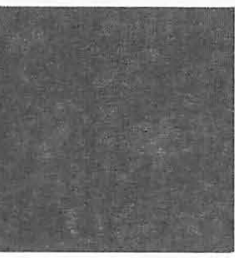

$\mathrm{k}=10$

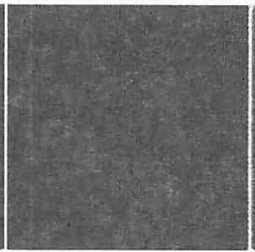

$\mathrm{k}=20$

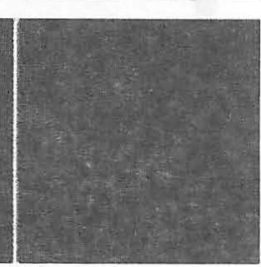

$\mathrm{k}=30$

Fig. 9 Detection by the adaptive threshold.

\section{Conclusion}

We proposed an adaptive threshold determination method for the RMF. The RMF enhances dim moving targets and makes it possible to detect them by thresholding. However, it is difficult to determine the threshold with a CFAR for actual images in advance. We showed that the output image of the RMF has a property in which the lower part of the histogram is invariant, regardless of whether there is a target or not. The proposed method exploits this property and estimates the mean and variance of the output noise from the output image directly. We also showed that CFAR is maintained by holding the normalized threshold constant. The threshold with CFAR is determined using the estimates and the normalized threshold. We evaluated the method by simulations and verified that the threshold with CFAR is obtained.

\section{References}

[1] Y. Barniv, "Dynamic programming solution for detecting dim moving targets," IEEE Trans. Aerosp. Electron. Syst., vol. AES-21, no. 1, pp. 144-156, 1985.

[2] J. Arnold, S. Shaw and H. Pasternack, "Efficient target tracking using dynamic programming," IEEE Trans. Aerosp. Electron. Syst., vol. AES-29, no. 1, pp. 44-56, 1993.

[3] S. D. Blostein and T. S. Huang, "Detecting small, moving objects in image sequences using sequential hypothesis testing," IEEE Trans. Signal Processing, vol. 39, no. 9, pp. 1611-1629, 1991.

[4] S. C. Pohlig, "An algorithm for detection of moving optical targets," IEEE Trans. Aerosp. Electron. Syst., vol. AES-25, no. 1, pp. 56-63, 1995.
[5] K. Nishiguchi and S. Yoshikawa, "Dim target tracking for star sensor using recursive maximum filter," 23rd International Symposium on Space Technology and Science, Matsue, Japan (2002)

[6] S. A. Mahmoud, M. S. Afifi and R. J. Green, "The effects of image background on velocity computation of moving objects," J. Microcomputer Applications, vol. 13, pp. 89-96, 1990.

[7] B. Porat and B. Friedlander, "A frequency domain algorithm for multiframe detection and estimation of dim targets," IEEE Trans. Pattern Anal. Machine Intell., vol. PAMI-12, no. 4, pp. 398-401, 1990.

[8] S. M. Tonissen, and R. J. Evans, "Performance of dynamic programming techniques for track-beforedetect," IEEE Trans. Aerosp. Electron. Syst., vol. AES-32, no. 4, pp. 1440-1451, 1996.

[9] L. Johnston and V. Krishnamurthy, "Performance analysis of a dynamic programming track before detect algorithm," IEEE Trans. Aerosp. Electron. Syst., vol. AES-38, no. 1, pp. 228-242, 2002.

[10] K. Nishiguchi et al., "Small target detection from image sequences using recursive max filter," SPIE Conference on Signal and Data Processing for Small Targets 1995, 2561, 153/166 (1995)

[11] K. Nishiguchi, "Performance analysis of recursive maximum filter," The 34th ISCIE International Symposium on Stochastic Systems Theory and Its Applications (SSS2002), pp. 29-35 (2003) 